\title{
O PAPEL DA VENTILAÇÃO LÍQUIDA NO TRATAMENTO DA INSUFICIENCIA RESPIRATÓRIA AGUDA EM CRIANÇS - UMA REVISÃO SISTEMÁTICA
}

Patrícia Freitas Góes*, Eduardo Juan Troster

Trabalho realizado no Instituto da Criança Professor Pedro de Alcântara da Universidade de São Paulo, São Paulo, SP

*Correspondência:

R. Passo da Pátria, 1407

Ap. 44 A2

Cep: 05085-000,

São Paulo, SP

pfgoes@ig.com.br

(11) $8264-1616 /(11)$

3833-9189

\begin{abstract}
RESUMO
OBjetivo. A ventilação líquida consiste no preenchimento total ou parcial dos pulmões por líquido (perfluorocarbono), eliminando a interface ar-líquido da membrana alveolar e reduzindo a tensão superficial em pulmões com deficiência de surfactante. Este trabalho tem como objetivo avaliar o papel da ventilação líquida no tratamento da insuficiência respiratória aguda em crianças.

Métodos. A técnica empregada foi uma revisão sistemática da literatura sobre ventilação líquida. O levantamento bibliográico utilizou os bancos de dados MEDLINE, LILACS, COCHRANE LIBRARY e referências de artigos. Os termos utilizados para pesquisa foram: liquid ventilation, respiratory distress syndrome, meconium aspiration syndrome, congenital diaphragmatic hernia e acute respiratory distress syndrome. Foram procurados ensaios clínicos randômicos, estudos de coorte, caso-controle e série de casos. Os desfechos avaliados foram resposta sobre a oxigenação sangưínea, mecânica respiratória e a sobrevida dos pacientes.
\end{abstract}

Resultados. Foram selecionadas 284 publicações relacionadas à ventilação líquida durante o período de estudo. Destas, 22 $(7,7 \%)$ eram ensaios clínicos, e apenas seis referiam-se à utilização da ventilação líquida em crianças. Todas as seis publicações foram caracterizadas como série de casos e analisadas separadamente.

Conclusäo. A partir da análise dos estudos clínicos, pôde-se concluir que, pela falta de estudos clínicos randômicos e controlados, a ventilação líquida não pode ser recomendada como terapia para insuficiência respiratória aguda em pediatria.

UnITERMOS: Insuficiência respiratória. Respiração artificial. Fluorocarbonetos. Alvéolos pulmonares. Crianças.

\section{INTRODUÇão}

Aidéia de redução máxima da tensão superficial tem sido explorada por meio da técnica de ventilação líquida (VL) utilizando compostos de perfluorocarbono.

A VL consiste no preenchimento total ou parcial dos pulmões por líquido, eliminando a interface ar-líquido da membrana alveolar e reduzindo a tensão superficial em pulmões com deficiência de surfactante. O líquido é administrado diretamente dentro dos pulmões através do tubo endotraqueal, recrutando alvéolos colapsados e facilitando a troca dos gases respiratórios. O objetivo da VL é facilitar a oferta de oxigênio e a remoção de gás carbônico, proteger os pulmões dos efeitos deletérios da ventilação mecânica convencional e promover a remoção de detritos pulmonares'.

A viabilidade da técnica de VL foi marcada após a descoberta dos compostos de perfluorocarbono. Estes são líquidos biologicamente inertes, transparentes, sem odor, derivados de compostos orgânicos comuns. Apresentam as seguintes propriedades que permitem a sua utilização no tratamento de pacientes com falência respiratória:

- alta solubilidade para o oxigênio e gás carbônico, melhorando a troca gasosa e reduzindo a toxicidade pelo oxigênio;

- alta densidade, permitindo alcançar regiões pulmonares dependentes e áreas pulmonares com atelectasias, recrutando alvéolos e aumentando a capacidade residual funcional. Esta característica reduz a lesão pulmonar causada por pressões inspiratórias elevadas e hiperdistensão alveolar observadas na ventilação mecânica convencional $1^{3,4}$;

- imiscibilidade com os fluidos corpóreos e água, ajudando na remoção de detritos pulmonares, além de evitar a lavagem e inativação do surfactante;

- baixa tensão superficial, permitindo ao perfluorocarbono melhorar a complacência pulmonar5;

- coeficiente de difusão positivo, permitindo dispersar-se de maneira ampla nos pulmões, recrutando áreas colapsadas e redistribuindo o fluxo sangüíneo pulmonar;

- efeito físico de barreira, retardando a formação de detritos e 0 extravasamento de células inflamatórias para o interior dos alvéolos;

- viscosidade semelhante à da água permitindo aos compostos de perfluorocarbono fluírem facilmente dentro dos pulmões;

- o vapor de pressão ideal para remoção rápida do perfluorocarbono via exalação;

- absorção mínima por meio dos alvéolos, além de não sofrerem metabolização no organismo, sendo eliminados do corpo por exalação por meio dos pulmões ou por evaporação por meio da pelé.

São descritas duas técnicas para a realização da VL: ventilação líquida total (VLT) e a ventilação líquida parcial (VLP) ou troca gasosa associada ao perfluorocarbono. 
Goes PF et AL.

Na VLT é instilado, por meio da cânula endotraqueal, um volume de perfluorocarbono igual à capacidade residual funcional de aproximadamente $30 \mathrm{ml} / \mathrm{kg}^{1,2,7}$. Alíquotas de volume corrente de líquido são, subseqüentemente, cicladas para efetivar a troca gasosa. O pulmão é completamente preenchido por líquido.

As investigações iniciais sobre VLT utilizavam um dos três métodos seguintes: imersão total do animal em um reservatório contendo líquido, a ventilação gravitacional assistida ou a ventiação mecanicamente assistida. Nos dois primeiros métodos, o líquido entrava e sáa dos pulmões sem assistência mecânica, acarretando aumento do tabalho respiratório e fadiga precoces. Na ventilação mecanicamente assistida, um aparelho propiciava assistência mecânica à respiração espontânea do animal. A necessidade de aparelhagem altamente especializada e complexa tem limitado a utilizaçãa da VL apenas em trabalhos experimentais?.

$\mathrm{Na}$ VLP os pulmões são parcialmente preenchidos por líquido (também equivalente à capacidade residual funcional). Posteriormente, um aparelho convencional de ventilação mecânica é acoplado e os pulmões são preenchidos por um volume corrente de gás ${ }^{8}$.

Durante a VLP, a capacidade residual funcional que permanece preenchida por líquido representa um reservatório incompreensível de oxigênio dissolvido, ocupando alvéolos que, de outra maneira, poderiam colapsar e permitir o shunt pulmonar. A VLP promove uma distribuição mais homogênea do líquido de perfluorocarbono por todas as regiões pulmonares, incluindo as regiões dependentes, mais comumente comprometidas, melhorando o recrutamento pulmonar nestas regiões. Ocorre também uma redistribuição do fluxo sangüíneo pulmonar para regiões não-dependentes, levando a uma melhora da oxigenação por meio da melhora da relação ventilação-perfusão nesta região, $0^{6,8}$.

Um grande número de estudos tem sugerido que a VLP é capaz de proteger os pulmões quanto ao desenvolvimento de lesões alveolares ou atenuar sua gravidade. Foi observada uma ação antiinflamatória espećíica do perfluorocarbono com inibição da produção de radicais-livres pelos macrófagos, diminuição na produção de citoquinas inflamatórias e diminuição do acúmulo de neutrófilos pulmonares após um agravo, contribuindo para redução da lesão pulmonar, ${ }^{9}$. Além disso, a VLP promove proteção contra o desenvolvimento de lesão pulmonar induzida pelo ventilador por meio da diminuição da tensão superficial, evitando a necessidade de altas pressões de vias aéreas e hiperdistensão alveolar!.

A VLP surge, portanto, como uma estratégia ventilatória para o tratamento da insuficiência respiratória grave. Esta técnica tem sido explorada em modelos animais por mais de três décadas.

Ensaios clínicos recentes têm mostrado a possibilidade de se manter a troca gasosa e a mecânica pulmonar adequadas em recémnascidos prematuros, crianças maiores e adultos submetidos à VL.

Esta revisão discute a experiência acumulada em estudos clínicos da técnica de VL, tentando estabelecer o seu papel no tratamento da insuficiência respiratória aguda de pacientes na faixa etária pediátrica.

\section{Métodos}

\section{Técnica empregada:}

O presente estudo consiste em uma revisão sistemática da literatura médica sobre VL.

\section{Identificação dos estudos:}

O levantamento bibliográfico foi feito de forma sistemática utillzando os seguintes bancos de dados:

- MEDLINE (período de 1966 a julho de 2005)

- LILACS (período de 198 I a julho de 2005)

- COCHRANE LIBRARY (período de 199I a julho de 2005)

- Referências encontradas em revisões, artigos e editoriais

Foram utilizadas publicações nos idiomas português, inglês e espanhol.

Os termos utilizados para a pesquisa foram os seguintes:

- liquid ventilation

- respiratory distress syndrome;

- meconium aspiration syndrome;

- congenital diaphragmatic hernia;

- acute respiratory distress syndrome.

\section{Critérios de seleção}

$\mathrm{Na}$ avaliação do tratamento da insuficiência respiratória, foram levados em consideração os seguintes critérios para seleção dos estudos:

\section{A) Desenho dos estudos}

- Ensaios clínicos randômicos

- Estudos de coorte

- Estudos tipo caso-controle

- Série de casos

\section{B)População}

Recém-nascidos, crianças e adolescentes até 18 anos com insuficiência respiratória aguda.

\section{C) Critérios de exclusão}

- Artigos de revisão não sistemática, capítulos de livros, editoriais e relatos de um único caso;

- Estudos realizados em animais de experimentação;

- Artigos publicados por um mesmo grupo de pesquisadores quando envolvessem a mesma população de estudo;

- Artigos que relatavam o uso da VL para outros fins;

- Artigos que inclúram adultos na população estudada.

\section{D) Tipo de intervenção}

Utilização de compostos de perfluorocarbono via cânula traqueal para tratamento de insuficiência respiratória aguda.

\section{E)Tipos de desfechos avaliados}

- Efeitos sobre a oxigenação sangǘnea avaliado por meio dos seguintes parâmetros:

1. Pressão arterial do oxigênio $\left(\mathrm{PaO}_{2}\right)$

2. Índice de oxigenação $(\mathrm{IO})$

3. Diferença alveólo-arterial de oxigênio $\left[\mathrm{D}(\mathrm{A}-\mathrm{a}) \mathrm{O}_{2}\right]$

- Efeitos sobre a complacência pulmonar

- Sobrevida dos pacientes submetidos à VL

\section{Resultados}

\section{Busca sistemática das publicações}

Foram selecionadas 284 publicações relacionadas à VL durante $\mathrm{O}$ período de estudo. Destas, 206 (72,6\%) estavam relacionadas a trabalhos envolvendo pesquisa experimental, 54 (19\%) constituíam-se 
de revisões não sistemáticas, editoriais e cartas, $2(0,7 \%)$ correspondiam a outras revisões sistemáticas e 22 (7,7\%) eram ensaios clínicos. Dos 22 ensaios clínicos identificados, apenas seis atendiam aos critérios de seleção estabelecidos, referindo-se à utilização da VL no tratamento da insuficiência respiratória aguda em crianças. Todas as seis publicações foram caracterizadas como séries de casos, não sendo encontrados ensaios clínicos randômicos. Uma das revisões sistemáticas selecionadas atendia aos critérios de inclusão, entretanto o único estudo envolvido na referida revisão não fora publicado, não podendo, portanto, ser utilizado para análise neste trabalho ${ }^{10}$.

\section{Ensaios clínicos}

\section{A) Estudo I - Greenspan et al., 1990"1}

O primeiro estudo clínico realizado em seres humanos utilizando compostos de perfluorocarbono foi realizado em 1990 por Greenspan et al. Este estudo teve como objetivo relatar os achados da utilização da $V L$ em recém-nascidos prematuros como uma terapia para melhorar a troca gasosa e a mecânica respiratória. Eles descreveram o uso da VLT por meio da técnica gravitacional assistida em três recém-nascidos prematuros com idade gestacional entre 23 e 28 semanas, refratários às técnicas convencionais de ventilação mecânica e considerados com risco elevado de mortalidade para que se justificasse uma terapia experimental. Foi demonstrada uma melhora importante na complacência pulmonar em todos os recém-nascidos estudados, além de melhora acentuada na oxigenação sangüínea em duas das três crianças envolvidas no estudo (Fig I). Após algumas horas de melhora mantida, as condições clínicas das crianças deterioraram, com evolução para óbito em até 19 horas depois de iniciada a VL. Este foi o primeiro e, até os dias atuais, o único relato de VLT em seres humanos.

\section{B) Estudo 2 - Gauger et al., 1996'2}

Gauger et al. avaliaram a utilização da VLP em seis pacientes pediátricos com síndrome de desconforto respiratório agudo que necessitaram de oxigenação de membrana extracorpórea.

A média da $\mathrm{PaO}_{2}$ aumentou de $39 \pm 6$ para $92 \pm 29 \mathrm{mmHg}$ durante as 96 horas após iniciada a primeira dose de perfluorocarbono $(p=0,021)$. A média da diferença alvéolo-arterial de oxigênio medida com $\mathrm{FIO}_{2}$ de $100 \%$ diminuiu de $635 \pm 10$ para $499 \pm 77 \mathrm{mmHg}$ durante o mesmo período $(p=0,059)$, enquanto a média da complacência pulmonar aumentou de 0,12 0,02 para 0,28 $\pm 0,08 \mathrm{ml} / \mathrm{cmH}_{2} \mathrm{O} / \mathrm{kg}$ $(p=0,0$ I $)$ (Fig 2). Todos os seis pacientes foram retirados com sucesso do suporte extracorpóreo e sobreviveram até a alta hospitalar.

\section{C) Estudo 3 - Pranikoff et al., 1996'13}

Pranikoff et al. avaliaram a segurança e a eficácia da VLP em quatro recém-nascidos com hérnia diafragmática congênita e insuficiência respiratória grave submetidos à oxigenação de membranas extracorpóreas.

Foram observados aumentos significantes na $\mathrm{PaO}_{2}(p=0,027)$ (Fig. 3), uma tendência ao aumento do conteúdo arterial de oxigênio $(p=0,052)$ e um aumento na complacência pulmonar $(p=0,007)$ (Fig. 4), após administração de dose diária de perfluorocarbono.

Todas as crianças foram retiradas com sucesso da oxigenação de membranas extracorpóreas e duas delas sobreviveram.

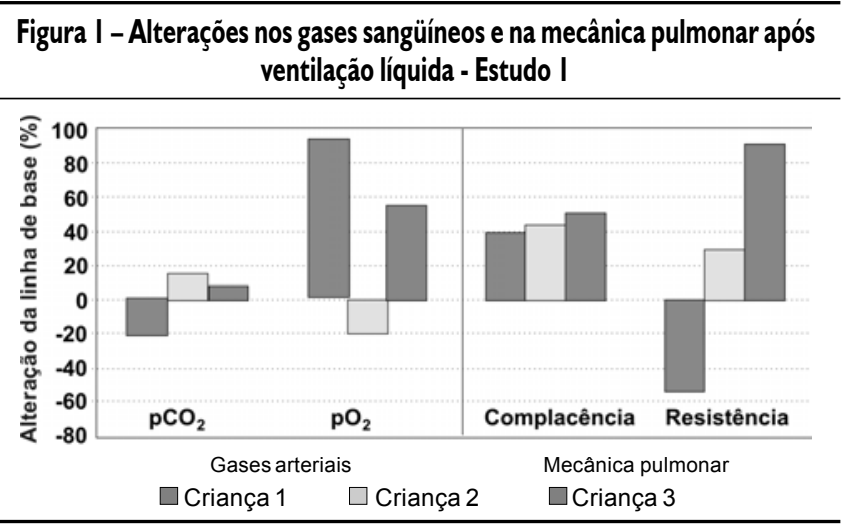

Figura 2 - Alterações na oxigenação e complacência pulmonar após ventilação líquida parcial - Estudo 2

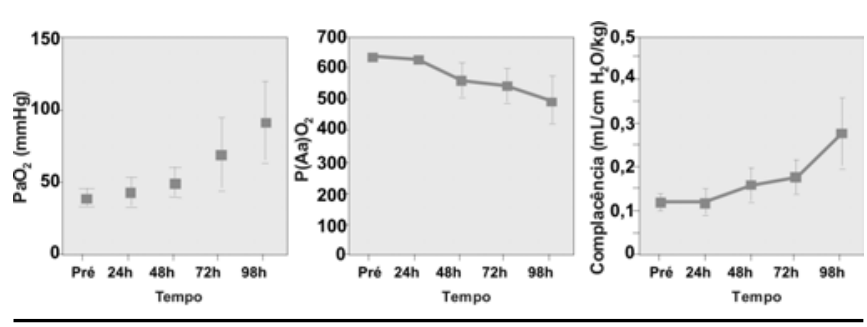

Figura $3-\mathrm{PaO}_{2}$ antes e após a administração de perfluorocarbono durante três dias de VLP - Estudo 3

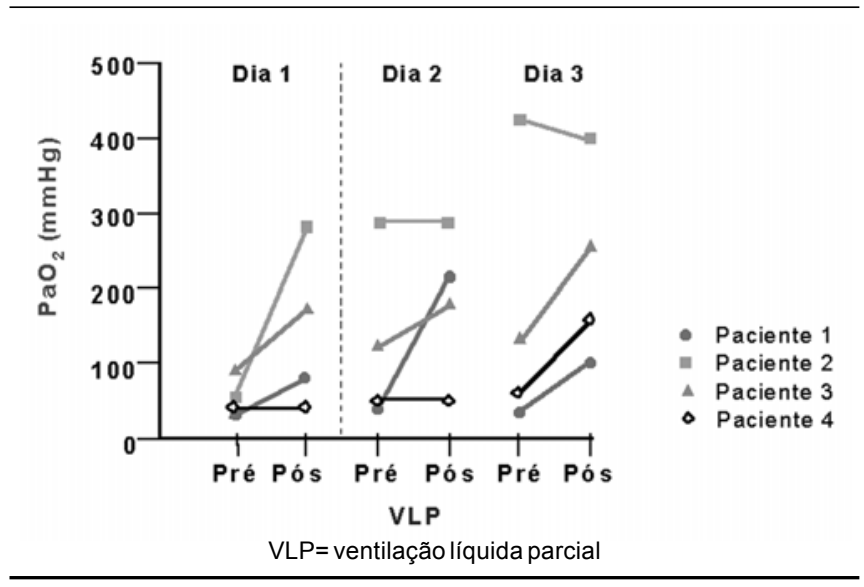

\section{D) Estudo 4 - Toro-Figueroa et al., 1996/4}

Os autores avaliaram a eficácia e segurança da VLP em dez crianças com idade entre I e 17 anos com diagnóstico de síndrome de desconforto respiratório agudo.

Nove de dez pacientes toleraram bem a instilação do perfluorocarbono. Um paciente piorou rapidamente sendo instalada a oxigenação de membranas extracorpóreas seis horas depois de iniciado o estudo. A troca gasosa melhorou em nove de dez pacientes (Tabela I). Duas mortes não associadas à VLP ocorreram nove e treze dias após o término do estudo. 
Figura 4 - Complacência pulmonar antes e após a administração de perfluorocarbono durante três dias de VLP - Estudo 3

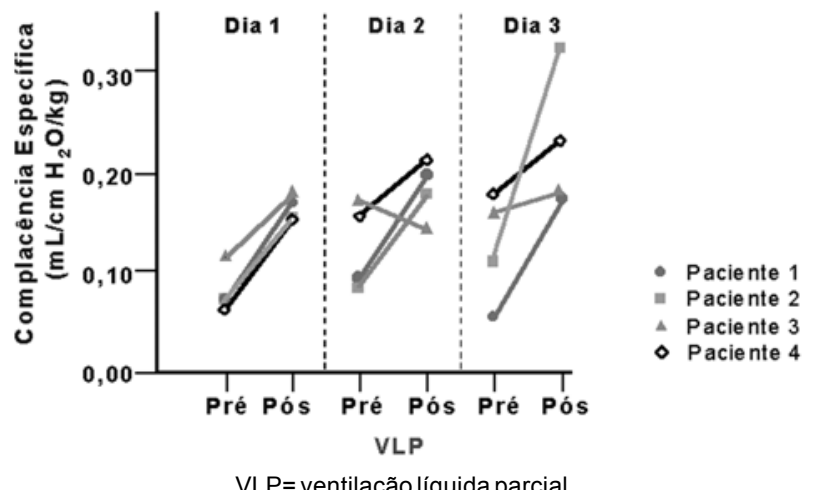

\begin{tabular}{|c|c|c|c|c|c|}
\hline \multicolumn{6}{|c|}{$\begin{array}{c}\text { Tabela I - Valores de } \mathrm{D}(\mathrm{A}-\mathrm{a}) \mathrm{O}_{2}, \mathrm{PaO}_{2}, \mathrm{PMVAS}, 10 \text { durante a } \\
\text { ventilação líquida parcial - Estudo } 4\end{array}$} \\
\hline & $\mathrm{n}$ & $\mathrm{D}(\mathrm{A}-\mathrm{a}) \mathrm{O}_{2}$ & $\mathrm{PaO}_{2} / \mathrm{FIO}_{2}$ & OMVA & 10 \\
\hline VCM & 10 & $457 \pm 42$ & $127 \pm 22$ & $20,6 \pm 1,7$ & $21,7 \pm 4,8$ \\
\hline 4 horas pós-VLP & 10 & $392 \pm 48$ & $144 \pm 29$ & $20,4 \pm 2,1$ & $22,5 \pm 7,1$ \\
\hline 8 horas pós-VLP & 9 & $322 \pm 49$ & $156 \pm 23$ & $18,8 \pm 1,3$ & $14,5 \pm 2,7$ \\
\hline 12 horas pós-VLP & 9 & $305 \pm 45$ & $148 \pm 20$ & $18,8 \pm 1,3$ & $15,7 \pm 2,9$ \\
\hline 24 horas pós-VLP & 9 & $286 \pm 46$ & $146 \pm 20$ & $18,0 \pm 1,5$ & $14,2 \pm 1,9$ \\
\hline 48 horas pós-VLP & 9 & $240 \pm 64$ & $199 \pm 42$ & $17,0 \pm 2,4$ & $|7,5 \pm 7|$, \\
\hline
\end{tabular}

VMC = ventilação mecânica; $V L P=$ ventilação líquida; $n=$ número;

$\mathrm{D}(\mathrm{A}-\mathrm{a}) \mathrm{O}_{2}=$ diferença alvéolo-arterial; $\mathrm{PaO}_{2}=$ pressão arterial de oxigênio;

PMVA = pressão média nas vias aéreas; 10 = índice de oxigenação

Figura $5-\mathrm{PaO}_{2}, \mathrm{PaCO}_{2}, \mathrm{FIO}_{2}$ e complacência média pulmonar durante ventilação a gás e ventilação líquida parcial - Estudo 5

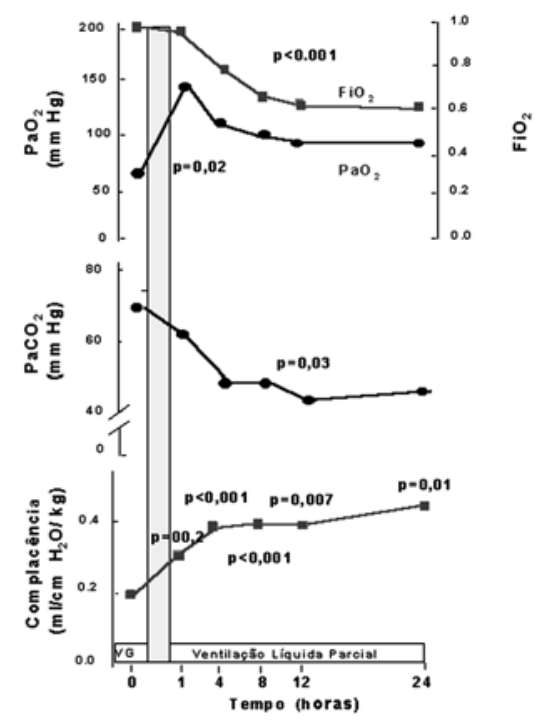

\section{E) Estudo 5 - Leach et al., $1996^{15}$}

O maior estudo realizado em pacientes pediátricos foi conduzido por Leach et al. envolvendo 13 recém-nascidos prematuros com síndrome de desconforto respiratório, refratária à terapia convencional. Os recém-nascidos tinham idade gestacional de 24 a 34 semanas e peso de nascimento de 600 a 2000g.

Dez crianças completaram o estudo. Uma hora depois de iniciada a $V L P$, a $P a O$, aumentou de $60 \pm 34 \mathrm{mmHg}$ para $143 \pm$ $99 \mathrm{mmHg}(p=0,02)$; o índice de oxigenação diminuiu de $49 \pm 60$ para $17 \pm 16(p=0,02)$; e a complacência aumentou de 0,18 \pm 0,12 para 0,29 $\pm 0,12 \mathrm{ml} / \mathrm{cm} \mathrm{H}_{2} \mathrm{O} / \mathrm{kg}(p=0,02)$ (Figura 5). Oito das 13 crianças, incluindo duas que foram retiradas do estudo, sobreviveram até uma idade gestacional corrigida de 36 semanas.

\section{F) Estudo 6 - Greenspan et al., 199716}

$\mathrm{O}$ objetivo deste estudo era demonstrar que um período de VLP com perfluorocarbono melhora a função pulmonar, sem efeitos colaterais, em um grupo de crianças recebendo oxigenação de membranas extracorpóreas com alta probalidade de morte. Seis crianças entraram no estudo, duas com hérnia diafragmática congênita e quatro com síndrome de desconforto respiratório agudo. Os dois pacientes com hérnia diafragmática morreram com disfunção pulmonar relacionada com a hipoplasia pulmonar e a hipertensão pulmonar grave. As duas crianças com síndrome de desconforto respiratório agudo que não sobreviveram morreram por problemas não relacionados à função pulmonar (falência de múltiplos órgãos devido à infecção herpética e doença intestinal dois meses e meio após a VLP).

Foi encontrada uma revisão sistemática da literatura que utilizou artigos do Registro Central de Estudos Controlados do Cochrane, Medline, CINAHL, jornais de terapia intensiva, conferências e listas de referência visando comprovar se a VLP reduz a morbi-mortalidade em crianças com lesão pulmonar aguda ou síndrome de desconforto respiratório agudo. Nesta revisão, foram pesquisados estudos randomizados e controlados que comparavam a VLP com outras formas de ventilação em crianças com lesão pulmonar aguda ou síndrome de desconforto respiratório agudo. Apenas um estudo envolvendo 182 pacientes (somente reportado como um abstract em uma conferência) foi incluído com base nos critérios de seleção. Este estudo foi encerrado prematuramente não tendo sido publicado e, portanto, sem condições de detectar diferenças significantes entre os métodos de ventilação estudados. A conclusão dos autores é que não existem estudos randomizados e controlados adequados e devidamente publicados que suportem o uso da VLPI0.

\section{Discussão}

O conceito da VL foi desenvolvido a partir dos princípios fisiológicos da função pulmonar. O preenchimento dos pulmões por líquido de perfluorocarbono diminui uniformemente a tensão superficial, apresentando melhora na complacência pulmonar. Como o líquido apresenta uma densidade maior que a do gás, pode recrutar alvéolos previamente colapsados, melhorando a capacidade residual funcional'. Os trabalhos experimentais das décadas de 1960 a 1980 conferem a plausibilidade biológica da técnica de $\mathrm{VL}$, demonstrando, portanto, a sua capacidade de melhorar a troca gasosa e a mecânica respiratória. 
A avaliação clínica dos benefícios da $\mathrm{VL}$ foi realizada por meio de estudos clínicos não randômicos e não controlados. $O$ único estudo randomizado não foi concluído, não tendo sido publicado. Os estudos encontrados descrevem o tratamento da doença em um grupo único de pacientes, em que todos recebem a intervenção em estudo. 0 pressuposto de tal abordagem é que, seja qual for a melhora após o tratamento, ela será atribuída à $V L$, o que não é necessariamente verdadeiro. Na falta de grupo-controle não é possível estabelecer uma relação causal entre tratamento em estudo e desfecho encontrado. Estes estudos, portanto, não têm o mesmo valor científico que os ensaios clínicos randômicos ${ }^{17}$.

Nesta revisão, foram encontrados seis estudos publicados que avaliaram a utilização de compostos de perfluorocarbono via cânula traqueal para tratamento da insuficiência respiratória aguda em recémnascidos, crianças maiores e adolescentes até 18 anos. Os estudos encontrados correspondiam a séries de casos, não controlados, não sendo possível agrupá-los para a realização de uma metanálise. Não foram realizadas análises estatísticas dos dados encontrados. Não existem dados baseados em evidências cientíícas, portanto, que justifiquem o uso clínico da VLP.

A VLP se mostrou uma técnica capaz de melhorar a mecânica respiratória e a troca gasosa em modelos com deficiência de surfactante. A ausência de evidências comprovando a eficácia da VLP não indica ausência de efeitos biológicos benéficos, sendo necessários novos estudos randômicos e com maior número de casos para melhor definir o seu papel no tratamento da insuficiência respiratória.

\section{Conclusão}

A partir da análise dos estudos clínicos, pôde-se concluir que, pela falta de estudos clínicos randômicos e controlados, a ventilação líquida não pode ser recomendada como terapia para insuficiência respiratória aguda em pediatria, até que novos dados sejam publicados.

\section{Conflito de interesse: não há.}

\section{SUMMARY}

THE ROLE OF LIQUID VENTILATION IN THE TREATMENT OF ACUTE RESPIRATORY FAILURE IN CHILDREN - A SYSTEMATIC REVIEW

BACKGROUND. Liquid Ventilation consists of partially or completely filling the lungs with a fluid (perfluorcarbon). This brings about elimination of the air-liquid interface and reduction of the surface tension in lungs with surfactant deficiency. This article focuses on the role of liquid ventilation in the treatment of acute respiratory failure in children.

METHODS. A systematic review of literature was conducted using MEDLINE, LILACS and COCHRANE LIBRARY databases and references of articles. Match terms were: Liquid Ventilation, Respiratory Distress Syndrome, Meconium Aspiration Syndrome, Congenital Diaphragmatic Hernia and Acute Respiratory Distress Syndrome. Randomized clinical trials, cohort studies and case control studies were searched for. Blood oxygenation, respiratory mechanics and survival were the outcomes evaluated.

RESULTS. Two hundred eighty four publications related to liquid ventilation were selected during the study period: 19(7.5\%) were clinical trials and only six of them focused on the use of Liquid Ventilation in children. All six publications were characterized as phase I and II clinical trials and analyzed separately.

CONCLUSIONS. Upon analysis of these trials it was concluded that, because of the lack of controlled and randomized clinical trials, liquid ventilation cannot be recommended as a therapy for children with acute respiratory failure. [Rev Assoc Med Bras 2006; 52(2): 103-7]

KEY WORDS: Respiratory failure. Artificial ventilation. Fluorocarbons. Pulmonary alveolii. Children.

\section{Referências}

I. Shaffer TH, Wolfson MR, Clark LC Jr. Liquid ventilation. Pediatr Pulmonol 1992; | 4: 102-9.

2. Leach CL, Fuhrman BP, Morin IFC, Rath MG. Perfluorocarbon-associated gas exchange (partial liquid ventilation) in respiratory distress syndrome: a prospective, randomized, controlled study. Crit Care Med 1993; 21:1270-8.

3. Hirschl RB, Pranikoff T, Wise C, Overbeck MC, Gauger P, Schreiner RJ, et al. Initial experience with partial liquid ventilation in adult patients with the acute respiratory distress syndrome. JAMA 1996; 275:383-9.

4. Hirschl RB, Tooley R, Parent A, Johnson K, Bartlett RH. Evaluation of gas exchange, pulmonary compliance, and lung injury during total and partial liquid ventilation in the acute respiratory distress syndrome. Crit Care Med 1996;24:1001-8.

5. Schwieler GH, Robertson B. Liquid ventilation in immature newborn rabbits. Biol Neonate 1976;29:343-53.

6. Shaffer TH, Wolfson MR, Greenspan JS, Hoffman RE, Davis SL, Clark LC Jr. Liquid ventilation in prmature lambs: uptake, biodistribution and eliminatios of perfluodecalin liquid. Reprod Fertil Dev 1996;8:409- I6.

7. Shaffer TH, Moskowitz GD. Demand-controlled liquid ventilation of the lungs. J Appl Physiol 1974;32:208- I3.

8. Fuhrman BP, Paczan PR, Defrancisis M. Perfluorocarbon-associated gas exchange. Crit Care Med 1991;19:712-22.

9. Rotta AT, Steinhorn DM. Partial liquid ventilation (PLV) attenuates oxidative damage to the lung in a model of acute lung injury (ALI). Crit Care Med 1998;26:Al I6(278).

10. Davies MW, Sargent PH. Partial liquid ventilation for the prevention of mortality and morbidity in paediatric acute lung injury and acute respiratory distress syndrome. Cochrane Library 2005; Issue 2.

II. Rotta AT, Steinhorn DM. Partial liquid ventilation reduces pulmonary neutrophil accumulation in a experimental model of systemic endotoxemia and acute lung injury. Crit Care Med I 998;26: I 707- I 5.

12. Greenspan JS, Wolfson MR, Rubenstein D, Shaffer TH. Liquid ventilation of human preterm neonates. J Pediatr 1990; I 17: I06-I I.

13. Gauger PG, Pranikoff T, Schreiner RJ, Moler FW, Hirschl RB. Initial experience with partial liquid ventilation in pediatric patients with the acute respiratory distress syndrome. Crit Care Med 1996;24:16-22.

14. Pranikoff T, Gauger PG, Hirschl RB. Partial liquid ventilation in newborn patients with congenital diaphragmatic hernia. J Paediatr Surg 1996; 31:613-8.

15. Toro-Figueroa LO, Meliones JN, Curtis SE, Thompson AE, Hirschl RB, Fackler JC, et al.. Perfluobron partial liquid ventilation (PLVP in chilfren with ARDS: a safety and efficacy pilot study. Crit Care Med I996;24:AI 50.

16. Leach CL, Greenspan JS, Rubenstein SD, Shaffer TH, Wofson MR, Jackson C, et al.. Partial liquid ventilation with perfluobron in premature infants with severe respiratory distress syndrome. N Engl J Med 1996;335:76 I-7.

17. Greenspan JS, FoxWW, Rubenstein SD, Wolfson MR, Spinner SS, Shaffer $\mathrm{TH}$. Partial liquid ventilation in critically ill infants receiving extracorporeal life support. Philadelphia liquid Ventilation Consortium. Pediatrics 1997;99:E2.

18. Fletcher RH, Fletcher SW. Epidemiologia clínica: elementos essenciais. Tradução de Maria Inês Schmidt. Porto Alegre: Artes Médicas; 1966.

Artigo recebido: 23/12/03

Aceito para publicação: 07/12/05 